\title{
UMA ABORDAGEM FUZZY PARA O ESTUDO DO PRESENTÉSMO
}

\section{A FUZZY APPROACH FOR THE STUDY OF PRESENTEEISM}

\author{
Recebido em 17.10.2016. Aprovado em 21.03.2017
}

Avaliado pelo sistema double blind review

DOI: http://dx.doi.org/10.12712/rpca.v11i2.830

\section{Kelly Marques de Oliveira Lopes}

kelly.unesp@yahoo.com.br

Universidade Estadual de Campinas (UNICAMP), Campinas/SP, BRASIL

Centro de Tecnologia da Informação Renato Archer (CTI Renato Archer) Amarais/SP, BRASIL

\section{Giovanna Garrido}

giovanna.garrido@cti.gov.br

Centro de Tecnologia da Informação Renato Archer (CTI Renato Archer) Amarais/SP, BRASIL

\section{Adriana Vazzoler Mendonça}

adriana.italia@gmail.com

Centro de Tecnologia da Informação Renato Archer (CTI Renato Archer) Amarais/SP, BRASIL

Anhanguera Educacional, Campinas/SP, BRASIL

\section{Marco Antonio Silveira}

marco.silveira@cti.gov.br

Faculdade de Campo Limpo Paulista (FACCAMP), Campo Limpo Paulista/SP, BRASIL

Centro de Tecnologia da Informação Renato Archer (CTI Renato Archer) Amarais/SP, BRASIL

\section{Resumo}

Este estudo tem como tema central um dos principais problemas relacionados ao capital humano: o Presenteísmo. Fundamentando-se na construção de um mapa sistêmico, que inclui as principais variáveis que circundam o construto, o objetivo do estudo é apresentar um modelo matemático Fuzzy para mensurar as relações entre elas e automatizar tal representação sistêmica. Para tanto, utiliza-se técnica de análise e julgamento para a tomada de decisão, gerando uma leitura quantitativa para as variáveis qualitativas representadas no mapa sistêmico. Justifica-se tal proposta pelo fato de que, por meio dela, torna-se possível a compreensão e a mensuração das relações causais e das influências recíprocas, direta ou inversamente proporcionais, entre as variáveis de interesse, oferecendo subsídios para decisões e servindo de orientação para as estratégias de amenização deste fenômeno que ameaça a sustentabilidade organizacional.

Palavras-chave: Lógica Fuzzy. Modelagem Matemática. Presenteísmo.

\begin{abstract}
This study has as central theme one of the main problems related to human capital: the Presenteeism. Basing on the construction of a systemic map, including the main variables that surround the construct, this research aims to present a mathematical model Fuzzy to measure the relationship between them and automatize this systemic graphic. Therefore, it is used a technique of analysis and judgment for decision-making in order to generate a quantitative reading for the qualitative variables that are represented in the systemic map. This proposal was judged pertinent for allowing the comprehension and measurement of the causal relationships and of the reciprocal influences, direct or inversely proportional, among variables of interest, offering subsidies to decision-making and guidelines for organizations to take action to reduce and prevent this phenomenon, which has been a threat for organizational sustainability.
\end{abstract}

Keywords: Fuzzy Logic. Mathematical Modeling. Presenteeism. 


\section{Introdução}

Uma vez que consiste no tema central deste estudo, é válida a exposição prévia do presenteísmo em termos conceituais: trata-se de uma combinação da ideia do indivíduo presente e do absenteísmo, denotando um comportamento característico de uma ausência invisível, em que o indivíduo está presente no trabalho, mas, ao mesmo tempo, ausente em mente ou comportamento de forma que a produtividade seja afetada.

Este comportamento tem sido foco de discussão nos ambientes organizacionais e acadêmicos, tendo em vista seus impactos negativos ao desempenho das organizações. Porém, esses debates limitamse a vieses analíticos que o relacionam apenas com aspectos da saúde do trabalhador, alegando ser um comportamento consequente do estar no trabalho doente. Costuma-se, portanto, explicar a consequente perda produtiva pela inadequação entre a capacidade do indivíduo ao trabalho, em termos físicos e mentais, e a exigência da tarefa.

Contudo, considera-se o presenteísmo como um comportamento que ultrapassa esta esfera. Assim, intuído em analisá-lo como algo não relacionado estritamente à doença, mas como algo crônico, o estudo parte de um direcionamento analítico que desvenda o presenteísmo na apropriação desequilibrada do tempo no trabalho com assuntos pessoais, fazendo menção à questão do não-trabalho no trabalho.

A análise do presenteísmo por meio desta abordagem estrutura-se na interpretação do fenômeno na interface entre fatores pessoais e fatores organizacionais, de modo que suas causas sejam atribuídas à propensão do trabalhador a esta postura ou mesmo à irracionalidade da própria organização, denotando o caráter voluntário e involuntário deste comportamento. Assim, por ser resultado de uma combinação complexa de fatores organizacionais e humanos, a identificação e medição do presenteísmo delimitam o maior desafio da gestão do capital humano, requerendo abordagens deste comportamento que vão ao encontro deste desafio e propiciem meios para mensurar seus impactos para o desempenho competitivo das empresas.

Deste modo, reconhecendo a relevância científica e organizacional de abordar o comportamento humano por meio de lógicas matemáticas, este estudo alinha- se a esse atual desafio da gestão constituindo uma abordagem Fuzzy para o estudo do presenteísmo.

Fundamentando-se na construção de um mapa sistêmico que inclui as principais variáveis que circundam o presenteísmo, o objetivo do estudo é apresentar um modelo matemático Fuzzy para mensurar as relações entre elas e automatizar tal representação sistêmica.

Justifica-se tal proposta pelo fato de que, por meio dela, torna-se possível a compreensão e a mensuração das relações causais e das influências recíprocas, direta ou inversamente proporcionais, entre as variáveis de interesse, oferecendo subsídios para decisões e servindo de orientação para as estratégias de amenização deste fenômeno que ameaça a sustentabilidade organizacional.

Para este fim, o artigo estrutura-se da seguinte forma: inicialmente, são demonstrados os procedimentos metodológicos adotados. Em seguida, delimita-se o referencial teórico subdividido em quatro tópicos principais. Primeiramente, é apresentado o conceito acerca do presenteísmo. Depois, fundamenta-se a questão do não-trabalho no trabalho como viés analítico do comportamento presenteísta, ressaltando a influência de fatores organizacionais e pessoais. Em seguida, as relações estabelecidas entre os principais fatores que circundam o presenteísmo são organizadas de maneira sistêmica. O quarto tópico traz a discussão central do artigo, apresentando um modelo matemático Fuzzy para mensurar as relações entre as variáveis qualitativas do comportamento presenteísta e automatizar as suas relações. Logo após, apresentam-se a análise dos dados e os resultados alcançados. Por fim, as considerações finais.

\section{Metodologia}

Com o intuito de apresentar um modelo matemático Fuzzy para automatizar a representação sistêmica do comportamento presenteísta, utiliza-se a técnica de análise e julgamento para a tomada de decisão, gerando uma leitura quantitativa para as variáveis qualitativas representadas no mapa sistêmico.

A abordagem do problema é caracterizada como qualitativa e quantitativa. Qualitativa, pois são estudadas as relações causais e as influências recíprocas entre as variáveis que circundam o comportamento presenteísta na empresa. Quantitativa, pois os 
resultados visam quantificar tais relações como variáveis de entrada e de saída, utilizando valores no intervalo entre $[0,1]$.

O trabalho de quantificação se desenvolveu a partir da construção de modelos matemáticos utilizando a Lógica Fuzzy. As Variáveis de entrada são apresentadas na Tabela 1 e as Variáveis de saída na Tabela 2.

Tabela 1. Variáveis de Entrada

\begin{tabular}{|l|c|c|c|}
\hline \multicolumn{4}{|c|}{ Variáveis de Entrada } \\
\hline Presenteísmo & Baixo & Médio & Alto \\
\hline Atenção & Baixa & Média & Alta \\
\hline Qualidade de Vida & Ruim & Regular & Boa \\
\hline Cooperação & Ruim & Regular & Boa \\
\hline
\end{tabular}

Fonte: Elaborada pelos autores

Tabela 2 . Variáveis de Saída

\begin{tabular}{|l|c|c|c|}
\hline \multicolumn{4}{|c|}{ Variáveis de Saída } \\
\hline Atenção & Baixa & Média & Alta \\
\hline Aprendizagem & Baixa & Média & Alta \\
\hline Produtividade & Baixa & Média & Alta \\
\hline Qualidade & Baixa & Média & Alta \\
\hline Presenteísmo & Baixo & Médio & Alto \\
\hline
\end{tabular}

Fonte: Elaborada pelos autores

O método de inferência utilizado foi o de Mamdani com a função Gaussiana, a qual, segundo Bede e Stefanini (2013), é a função mais utilizada para controladores Fuzzy.

\section{Referencial teórico}

\section{Presenteísmo: uma delimitação do conceito}

No que tange à área do comportamento organizacional, os esforços pela diminuição dos problemas associados ao capital humano tornam-se uma prioridade. Atenção considerável tem sido direcionada ao absenteísmo e ao presenteísmo. Em virtude de seus impactos para o desempenho econômico e operacional das organizações, para os trabalhadores e para a sociedade, frequentes têm sido os estudos das causas e decorrências concernentes a eles.

Apesar de consistir em um fenômeno cujas manifestações vão além daquilo que é percebido e mensurável, constata-se que o presenteísmo se porta como um mal ainda mais pernicioso que o absenteísmo. Mesmo de forma silenciosa, o comportamento presenteísta tem sido considerado um fator de alto custo e forte impacto na produtividade, uma condição negativa ao resultado econômico das organizações (D'ABATE e EDDY, 2007; UMANN; GUIDO; GRAZZIANO, 2012).

Vale dizer que, diferentemente da consistência das considerações de seus impactos na produtividade, observa-se que os debates acerca do fenômeno limitam-se a vieses analíticos que o relacionam apenas com aspectos da saúde do trabalhador. Grande parte dos estudos sobre o presenteísmo enviesa a perda de produtividade como algo estritamente relacionado à doença, alegando ser consequência da inadequação entre a capacidade do indivíduo ao trabalho, em termos físicos e mentais, e a exigência da tarefa (BÖCKERMAN e LAUKKANEN, 2009, 2010; COLLINS e CARTWRIGHT, 2012; FERREIRA et al., 2015; TALOYAN et al., 2012; UMANN; GUIDO; GRAZZIANO, 2012; UMANN; GUIDO; SILVA, 2014).

Assim, com o intuito de estender o construto para além desta esfera, assume-se a conceituação estabelecida por D'Abate e Eddy (2007) que, combinando a ideia do indivíduo presente e do absenteísmo, definem o presenteísmo como o estar presente no trabalho, mas ausente em mente ou comportamento, de forma que a produtividade seja afetada. Isto é, um comportamento característico de uma ausência invisível. Tal escolha se deve à capacidade do enunciado de sintetizar o teor analítico pretendido neste estudo: o presenté́smo como algo que não tem relações com doença, mas como algo crônico.

Sendo assim fundamentado, o caráter irrestrito do estudo às relações entre o presenteísmo e a saúde do trabalhador direciona a interpretação deste comportamento para um viés analítico que desvenda a ação presenteísta na apropriação desequilibrada do tempo no trabalho com assuntos pessoais, fazendo menção à questão do não-trabalho no trabalho. 


\section{Não-trabalho no trabalho: uma apropriação silenciosa do tempo}

Conforme o exposto no tópico 3.1, o teor analítico deste estudo consiste em abordar o presenteísmo como um comportamento que não é estritamente relacionado à saúde do trabalhador, mas que pode assumir um caráter crônico, independentemente da condição física daquele que o manifesta. Para tanto, constitui-se uma abordagem que desvenda a manifestação deste comportamento no envolvimento em não-trabalho no trabalho. É válido afirmar que a razão pela qual o estudo alinha a etiologia do presenteísmo à questão do não-trabalho no trabalho se deve ao fato de as atividades não relacionadas ao trabalho e o desvio no trabalho já se estabelecerem como sinônimos do presenteísmo (EDDY; D'ABATE; THURSTON JR, 2010).

Sabe-se que os indivíduos gerenciam uma gama de papéis relacionados ao trabalho e ao não-trabalho. Sua atenção é dividida para o atendimento de três tipos de demanda: trabalho, família e lazer. A questão é que tais demandas não se portam como entidades de gerenciamento individualizadas. Delimitadas por fronteiras permeáveis, muitas das vezes, elas se sobrepõem umas às outras. Assim, mesmo que fisicamente engajado no gerenciamento das demandas de um papel, o indivíduo pode não permanecer focado e não opera somente para este fim. É natural que, em mente ou comportamento, ele transponha as fronteiras que o separam dos demais papéis (D'ABATE, 2005; EDDY; D'ABATE; THURSTON JR., 2010; VIJAYAKUMAR, 2015).

Esta convergência entre as demandas da vida pessoal e do trabalho foi propiciada pelo próprio desenvolvimento tecnológico. É fato que, quando se trata de uma sobreposição e não de uma competição entre as demandas, tal ocorrência não é maléfica. Afinal, o equilíbrio entre os vários domínios da vida é importante e possível de ser alcançado (D'ABATE, 2005; IVARSSOM e LARSSOM, 2012; VIJAYAKUMAR, 2015).

O problema reside quando o indivíduo nota um desequilíbrio em seus papéis. São frequentes os casos em que, por interferências internas ou externas, um domínio passa a exercer supremacia e requerer atenção e recursos a ponto de criar conflito perante os demais (VIJAYAKUMAR, 2015). A consequência disso é o desbalanceamento do esforço emocional e da energia requeridos no atendimento das demandas presentes, refletindo no rendimento individual (D'ABATE, 2005).
Éa incorporação dos três domínios da vida que sustenta este debate sobre o comportamento presenteísta, alinhando a sua ocorrência ao envolvimento do trabalhador em assuntos não relacionados ao trabalho no trabalho, de modo que se tenha uma apropriação desequilibrada do tempo, do esforço, da inclinação e da energia despendida no atendimento às demandas do trabalho. Daí seu impacto na produtividade (D'ABATE e EDDY, 2007).

Vale dizer que a apropriação do tempo no trabalho não provém apenas de fatores pessoais. Muitas das vezes, fatores organizacionais induzem esta postura. Seja por questões estruturais, organizacionais e institucionais (PAULSEN, 2015), pelo ambiente organizacional suscetível à postura (EDDY; D'ABATE; THURSTON JR, 2010; PAGE, 2015), ou mesmo por razões mais implícitas, como uma forma de lidar com o estresse (PAGE, 2015), a busca pelo prazer e pelo equilíbrio almejado (D'ABATE, 2005; D'ABATE e EDDY, 2007; EDDY; D'ABATE; THURSTON JR, 2010; PAGE, 2015), por insatisfação, por tédio (D'ABATE, 2005; PAGE, 2015; SAARVALA, 2006; VIJAYAKUMAR, 2015), pela tendência a procrastinação (D'ABATE e EDDY, 2007) e pela própria falta de sentido naquilo que é feito (D'ABATE, 2005; PAGE, 2015), o trabalhador passa a estar presente no trabalho, mas ausente em mente, em comportamento, em capacidade para realizar as suas tarefas de forma produtiva e eficaz. Isto é, como um meio de reduzir sua própria frustração e descontentamento e de satisfazer suas necessidades e vícios psicológicos, ele apropria silenciosamente seu tempo em outras coisas, simulando, disfarçando e sabotando a sua própria produtividade (FERREIRA e ESTEVES, 2016; PAULSEN, 2015).

Isso faz da postura presenteísta um dos principais problemas organizacionais relacionados ao capital humano. Porém, é importante notar que este comportamento é consequência da própria noção organizacional que desvaloriza o capital humano. É notória a persistência de sistemas organizacionais que absorvem a personalidade e colonizam a subjetividade, não permitindo nenhuma manifestação de resistência. Isso acaba fazendo com que os indivíduos adotem formas mais sutis de resistência (PAGE, 2015; PAULSEN, 2011), como uma espécie de decaf resistance, assumindo posturas que a simbolizam, mas não testemunham os limites que gera e a violência que a envolve, sendo apenas submersas no silêncio (CONTU, 2008). 
Além disso, a ação presenteísta de apropriar o tempo de trabalho em não-trabalho e ter a produtividade prejudicada não se define apenas como uma ação de resistência. Muitas vezes, o indivíduo pode ser forçado à postura pelo próprio desperdício organizado, pela cultura presenteísta que permeia a organização, tendo em vista as explosões de descontentamento entre os trabalhadores, que levam ao tédio e à alienação (PAGE, 2015; PAULSEN, 2011, 2015).

Ao delimitar uma tipologia representativa da apropriação do tempo no trabalho combinando fatores relacionados à inclinação do indivíduo ao trabalho e fatores relacionados às exigências da tarefa, Paulsen (2015) subsidia este debate acerca da influência de fatores organizacionais e pessoais no presenteísmo. A interpretação de sua obra permite inferir que, combinando eixos representativos da subjetividade do trabalhador e da objetividade do trabalho, delimitamse contextos em que o comportamento presenteísta é resultante de fatores pessoais e contextos em que a própria irracionalidade da organização do trabalho o determina, denotando o caráter involuntário deste comportamento. Isto é, quando a opacidade na definição de cargos e tarefas, em termos de suas exigências e da capacidade de seus executores, propicia a conduta presenteísta (PAULSEN, 2011, 2015).

Vale enfatizar que, referindo-se à inclinação individual ao trabalho, Paulsen (2015) afirma que o seu teor não provém da gestão, mas do significado do trabalho. Ou seja, esta inclinação é um fenômeno endógeno que depende da forma como o indivíduo enxerga o trabalho que executa.

Aliás, conforme é debatido na literatura (EDDY; D'ABATE; THURSTON JR, 2012; FRITZ et al., 2013; FRITZ; LAM; SPREITZER, 2011; PAGE, 2015; PAULSEN, 2011, 2015, entre outros), são fortes as ligações entre o comportamento presenteísta e o significado que o indivíduo atribui ao trabalho, tornando-o mais agudo quanto menor for este significado.

De qualquer modo, a motivação para estas apropriações indevidas do tempo no trabalho são diversas. Ora elas aparecem como um meio de indignação pessoal, ora política, quando implícita à empresa e explícita entre os trabalhadores como uma forma de provocar mudanças, ou mesmo como resultado de algo já introjetado na cultura da empresa. Muitas vezes, pela forma como o trabalho é organizado, a simulação torna-se tão integrada à tarefa que agir de maneira diferente é como um suicídio organizacional, induzindo o resigno passivo como um ajuste ao próprio desperdício (PAULSEN, 2011).

Paulsen (2011) também pontua que a própria resignação individual em alegar necessidade de tempo livre motiva a postura. Sabe-se que os indivíduos não se mantêm ativos e engajados em suas atividades todo o expediente. Embora eles difiram entre si pela forma como criam fronteiras entre o trabalho e o não-trabalho, a própria necessidade de sustentação de energias induz microparadas para alívio de fadiga e desconforto físico. Aliás, existem alguns estudos que afirmam que microparadas durante o trabalho são vistas como positivas. Alega-se que o distanciamento mental e psicológico do trabalho, através do envolvimento com outros assuntos, é uma forma de manter ou repor recursos psicológicos considerados limitados, porém importantes para o desempenho (FRITZ et al., 2013; FRITZ; LAM; SPREITZER, 2011).

Deste modo, enfatiza-se que a relação benéfica entre distanciamento mental e psicológico e desempenho no trabalho depende do nível em que ela ocorre. Isto é, níveis muito altos ou baixos de distanciamento são prejudiciais para o desempenho. Assim, é necessário moderação no envolvimento em não-trabalho no trabalho, o suficiente para a recuperação de energias (FRITZ et al., 2013; VIJAYAKUMAR, 2015).

Neste ponto, destaca-se o papel do significado do trabalho nesta tendência presenteísta de apropriação de tempo no trabalho. Quanto maior o significado que o indivíduo atribui ao seu trabalho, menor é a sua necessidade de se desapegar dele para reposição de energias (FRITZ et al., 2013; VITAYAKUMAR, 2015). Considerando o estado afetivo do indivíduo como um recurso para o trabalho, sendo identificado com o que faz, não é o fato de se distanciar mental ou psicologicamente que garantirá seu bem estar e satisfação. O trabalho em si já é uma fonte de prazer (TROUGAKOS e HIDEG, 2009).

Cruciais para a pretensão deste estudo são os pareceres de Fritz, Lam e Spreitzer (2011) e Trougakos e Hideg (2009) de que é a natureza e o conteúdo do distanciamento mental e psicológico do trabalho que determinam o teor da recuperação de energias e o desempenho individual. Isto é, microparadas no trabalho só são positivas dependendo do tipo de atividade realizada no momento (FRITZ et al., 2013). Assim, constata-se que envolver-se em não-trabalho como um meio de recuperação de recursos psicológicos 
não está associado à manutenção ou reposição de energias (FRITZ; LAM; SPREITZER, 2011).

Trata-se, portanto, de uma estratégia popular e frequente que acaba subsidiando e induzindo o caráter crônico do comportamento presenteísta. Seja por apatia ou como uma manifestação de resistência, a opção por se envolver em não-trabalho no trabalho não retribui ao indivíduo os efeitos desejados, sejam em méritos de reposição de energias ou mesmo de um estado afetivo positivo. Assim sendo, este comportamento torna-se um mal crônico que, atenuando o capital humano, silenciosamente impacta o desempenho organizacional em todos seus aspectos.

Como se pode observar, a manifestação do comportamento presenteísta é atrelada a uma combinação complexa de fatores pessoais e organizacionais. Assim, a identificação e medição deste comportamento delimitam o maior desafio da gestão, requerendo abordagens que propiciem meios para mensurar seus impactos para o indivíduo e para o desempenho competitivo das empresas.

É em consonância a este desafio que o estudo se propõe a abordar o presenteísmo recorrendo à Lógica Fuzzy, assumindo como fundamento o mapa descrito a seguir.

\section{A postura presenteísta: causas e efeitos sistemicamente representados}

Visando a representação gráfica do presenteísmo na interface de fatores pessoais e organizacionais e a fundamentação do modelo Fuzzy proposto neste estudo, na Figura 1 é demonstrada uma versão simplificada de um mapa sistêmico que organiza os principais fatores que circundam este comportamento, denotando a causalidade estabelecida entre eles e seus efeitos na produtividade do trabalho e no desempenho das organizações.
Figura 1. Mapa sistêmico

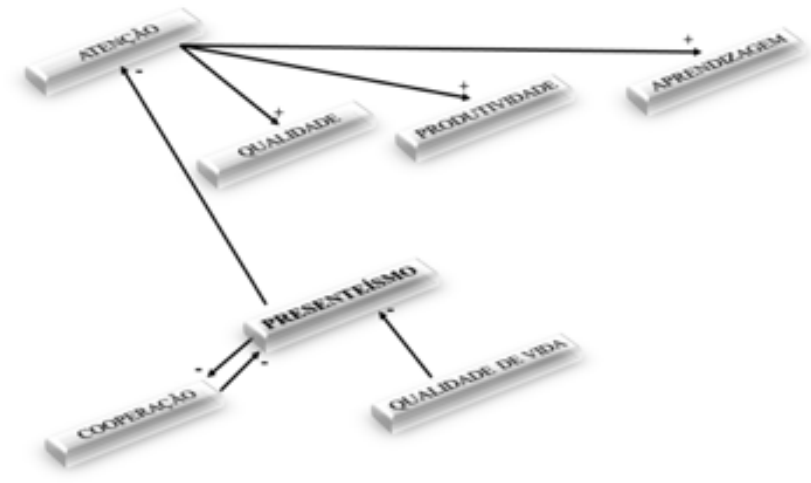

Fonte: Elaborada pelos autores

Observa-se que o mapa é composto pelos seis principais fatores relacionados com a variável Presenteísmo, a saber: Qualidade de Vida, Cooperação, Atenção, Qualidade, Produtividade e Aprendizagem.

O fundamento de tal representação, assim como de todos os mapas sistêmicos, tem como base hipóteses, intuições e o próprio conhecimento especializado acerca das relações recíprocas estabelecidas entre os fatores. Vale a explicação de que as relações expostas são diretamente proporcionais, quando representadas com o sinal (+), e inversamente proporcionais, quando representadas com o sinal (-).

Assim, este mapa representa uma sumarização das questões discutidas a respeito do comportamento presenteísta a partir de sua acepção de não-trabalho no trabalho. Concernentes a sua posição no mapa principal, observa-se que o comportamento tem como antecedentes principais a Qualidade de Vida e a Cooperação.

A relação inversamente proporcional entre Presenteísmo e Qualidade de Vida explicita que a apropriação desequilibrada do tempo no trabalho se deve à presença de elementos estressores e a outros indicadores físicos e psicológicos de bem estar (VIJAYAKUMAR, 2015).

A Cooperação também consiste em um fator inversamente relacionado ao Presenteísmo. Pela sua influência no estresse e na consequente alteração de humor e desmotivação, a falta de atitudes cooperativas se estabelece como razão do comportamento presenteísta (SAARVALA, 2006). A qualidade do relacionamento e da cooperação no trabalho atua na reposição dos recursos psicológicos e quanto mais energizado física e psicologicamente, menor é a 
propensão individual em ausentar-se do trabalho em mente ou comportamento e apropriar o tempo em não-trabalho (FRITZ; LAM; SPREITZER, 2011).

Nota-se que a relação entre Cooperação e Presenteísmo é biunívoca. Assim, a apropriação do tempo no trabalho com não-trabalho também impacta nas atitudes cooperativas e no relacionamento interpessoal. Mesmo que presente, o distanciamento mental e comportamental e a falta de foco no trabalho impossibilitam o gerenciamento das demandas e atitudes concernentes a ele.

Já os efeitos do Presenteísmo no resultado organizacional são delimitados pelo seu impacto no índice de Atenção individual, fator diretamente relacionado com a Produtividade, Qualidade e Aprendizagem. Isto é, pelos lapsos de atenção que gera, o presenteísmo limita o desempenho em quantidade, pela diminuição do rendimento físico e mental, em qualidade, pela possibilidade de erros no desenvolvimento das atividades laborais (UMANN; GUIDO; GRAZZIANO, 2012) e em méritos de capacitação, visto que, estando engajado em nãotrabalho, menor é o tempo despendido pelo indivíduo no desenvolvimento de novos conhecimentos e habilidades. Como visto, a falta de atenção é o que dificulta o desempenho eficiente e eficaz no trabalho (D'ABATE e EDDY, 2007).

Em suma, comprova-se a opinião de Böckerman e Laukkanen (2010) de que o presenteísmo tem como determinantes tanto as características do trabalhador como do trabalho. Isto é, sob influência de fatores pessoais e organizacionais, tem-se um comportamento que, subterraneamente, ameaça a sustentabilidade organizacional. Ciente de que o conceito de sustentabilidade se resume na combinação entre Qualidade de Vida e resultado organizacional (SILVEIRA e BECARO, 2014), corrompida esta combinação, silenciosos e cruéis são seus efeitos no âmbito organizacional.

Sabe-se que, desde os primórdios das relações de emprego, a organização e o trabalhador participam de uma relação simbiótica, em que um não existe sem o outro e que o comportamento de um modifica o comportamento do outro. Deste modo, pode-se considerar que a partir do momento em que a organização disponibiliza boas condições de trabalho para o trabalhador, maiores são as chances de o trabalhador responder com enriquecimento do capital humano disponibilizado para a organização
(SILVEIRA et al., 2015), sendo evidente que menores são as tendências presenteístas.

Em suma, a abordagem do presenteísmo na interface de determinantes pessoais e organizacionais sinaliza que a proposta deste estudo reside em utilizar o potencial da lógica Fuzzy como um meio de compreender e mensurar as relações entre essas variáveis de interesse, de modo a subsidiar estratégias que alinhem os interesses dos trabalhadores e os da organização e, com isso, amenizem esse mal.

Assim, no tópico seguinte serão apresentados os fundamentos da Lógica Fuzzy e o que faz dela um modelo matemático potencial para a aplicação proposta neste estudo.

\section{A aplicação da lógica Fuzzy}

A Lógica Fuzzy tem por objetivo representar a imprevisão, ambiguidade e vagueza do raciocínio humano. Fornecendo ferramentas capazes de medir variáveis qualitativas, ela se mostra capaz de modelar o senso de palavras, a tomada de decisão ou até mesmo o senso comum do ser humano (BARROS e BASSANEZI, 2010).

A teoria de Lógica Fuzzy, também conhecida como Lógica Nebulosa, foi desenvolvida pelo professor Zadeh (1965), com a publicação do artigo Fuzzy Sets no Jounal Information and Control. Sua finalidade era conseguir controlar máquinas à vapor e possibilitar a modelagem matemática das ações dos operadores de máquinas, automatizando suas tarefas.

Ao contrário da Lógica Booleana, a Lógica Fuzzy não trabalha com os conceitos de verdadeiro ou falso, mas permite uma análise não tão precisa com relação às premissas. Por exemplo, a variável pessoa não precisa ser dividida apenas em magra ou gorda. De acordo com a Lógica Fuzzy, pode-se dividir em: extremamente magra, pouco magra, magra, pouco gorda, gorda e extremamente gorda. Isto é, a Lógica Fuzzy permite uma série de possibilidades para as análises das variáveis.

A proposta de Zadeh (1965) foi de considerar uma função que forneça um grau de pertinência variando entre $[0,1]$. Quando o grau de pertinência for igual a 0 , tem-se uma pertinência nula. Quando for igual a 1, tem-se uma pertinência total (SHAW e SIMÕES, 1999).

Essa transição do verdadeiro ou falso da Lógica Booleana para o intervalo $[0,1]$ possibilitou a criação 
das variáveis linguísticas, permitindo explorá-las e compará-las com o raciocínio humano.

Inicialmente, a Lógica Fuzzy não foi muito aceita por conta dos matemáticos tradicionais que não dispensavam a Lógica Tradicional. Mas, diante da possibilidade do tratamento de dados não precisos, a Lógica Fuzzy foi sendo rapidamente desenvolvida, principalmente no Japão, onde foi criado o primeiro grupo de pesquisa em Sistema Fuzzy em 1984 (SHAW e SIMÕES, 1999).

Segundo Yen, Langari e Zadeth (1994), a lógica tem um vasto campo de aplicações, tais como na automatização de máquinas, no controle de câmeras fotográficas, nos sistemas de acionamento de robôs, na otimização de processos produtivos, entre outros.

Dessa forma, a Lógica Fuzzy é definida por Cox (1995) como sendo a combinação de eventos naturais incertos juntamente com a lógica das máquinas, resultando em respostas robustas, inteligentes e flexíveis.

Para Gupta e Kaufmann (1988), a lógica é formada por técnicas que interpretam o raciocínio humano. $\mathrm{O}$ processo de análise das variáveis é chamado de Sistema Lógico Fuzzy e pode ser representado conforme a Figura 2.

Figura 2 . Sistema Lógico Fuzzy

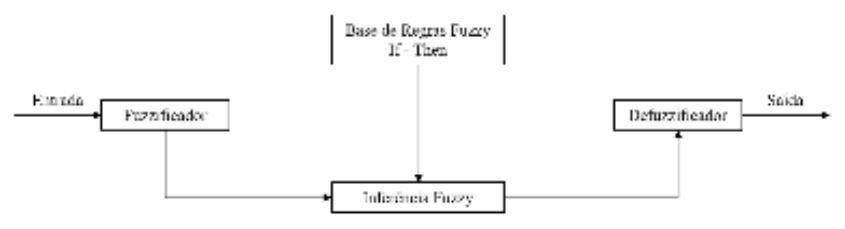

Fonte: Cox (1995)

No cotidiano do ser humano, existem muitos problemas que são impossíveis de responder com precisão, sendo necessário considerá-los com certa flexibilidade. Diante destes fatos, tratando-se de problemas relacionados à natureza humana, a Lógica Fuzzy tem se mostrado bastante adequada. Afinal, a mente humana possui elementos ilógicos, como sentimentos e intuições, que são impossíveis de mensurar através de um formalismo matemático tradicional.

Segundo Silveira et al. (2004), a Lógica Fuzzy auxilia na gestão de alocação de recursos, auxiliando o gestor a administrar de forma mais eficiente os ambientes de trabalho com alto índice de competitividade. Para os autores, a Lógica se porta como uma ferramenta para as incertezas do meio organizacional.

Para Cox (1995), a Lógica Fuzzy trabalha com uma métrica imprecisa que, quando aplicada a certos tipos de problemas, consegue traduzi-los de uma forma mais real, ou mais próximo da realidade.

A utilização da lógica fuzzy
em problemas de avaliação de
fenômenos agrários, biológicos
e administrativos está tornando-
se uma alternativa extremamente
eficiente e eficaz frente a métodos
estocásticos recorrentes. Contudo,
apesar de já serem observados
estudos desenvolvendo modelos
matemáticos de lógica fuzzy
aplicados na redução do impacto
da tecnologia e otimização dos
processos produtivos, ainda são
discretas as iniciativas de aplicação
destes modelos na área de
recursos humanos da organização,
responsável pelos planos de cargos,
e por delimitar as habilidades e
competências requeridas para o
trabalho (GABRIEL e LUÍS, 2012,
395).

Moreira e Baptista (2012) ressaltam que as mudanças tecnológicas estão influenciando nos processos de Recursos Humanos das empresas, exigindo novos modelos de trabalho e de novos trabalhadores.

\section{Resultados e análises dos dados}

Para a automatização do Mapa Sistêmico (Figura 1), utilizou-se a Lógica Fuzzy com a inferência de Mamdani e a função Gaussiana. O estudo dividiu o problema em três casos: $1^{\circ}$ ) Como o Presenteísmo interfere na Atenção; $2^{\circ}$ ) Como a Atenção interfere na Aprendizagem, Produtividade e na Qualidade; e $3^{\circ}$ ) Como a Qualidade de Vida e a Cooperação interferem no Presenteísmo.

A atribuição de termos linguísticos em referência ao nível de cada uma das variáveis abordadas e de 
funções de pertinência do tipo Gaussiana permitiu o estabelecimento de regras linguísticas para cada um dos casos, tendo como base a análise do conjunto dos dados que envolvem as variáveis abordadas.

Para o Caso $1^{\circ}$, pode-se observar na Figura 3 o nível da variável Presenteísmo e da variável Atenção.

Figura 3 . Nível da variável de entrada Presenteísmo e da variável de saída Atenção
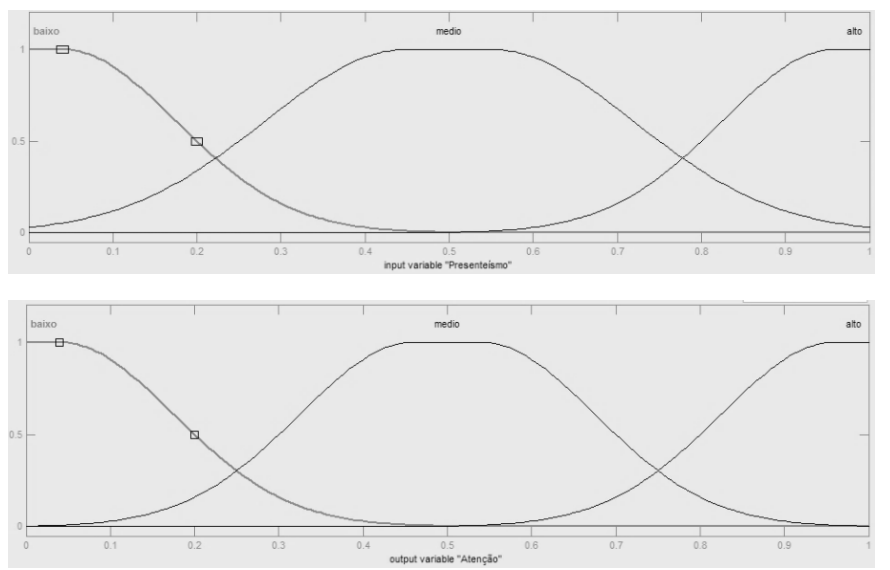

Fonte: Elaborada pelos autores

Como se nota, os pontos de máximo e de mínimo global e local da função estão em torno de $(0,0.84)$ e $(1,0.16)$. Ou seja, se o Presenteísmo for baixo, a Atenção será máxima, em torno de $84 \%$. Em contrapartida, se o Presenteísmo estiver no seu valor máximo igual a 1 , a Atenção terá seu valor mínimo em torno de 16\%.

Expõe-se a visão geral do comportamento e da variação das variáveis estudadas no Caso $1^{\circ}$ na Figura 4, na qual é demonstrado o comportamento da variável Atenção em relação aos valores de mínimo e de máximo da variável Presenteísmo.

Figura 4 . Gráficos de saída das variáveis Presenteísmo

$$
\text { e Atenção }
$$

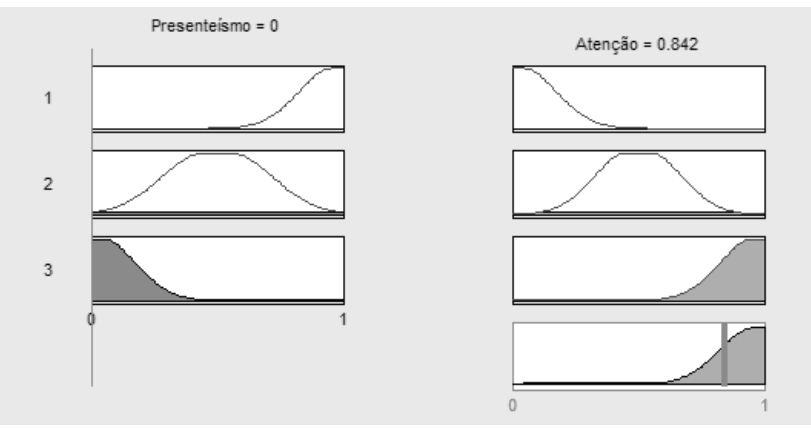

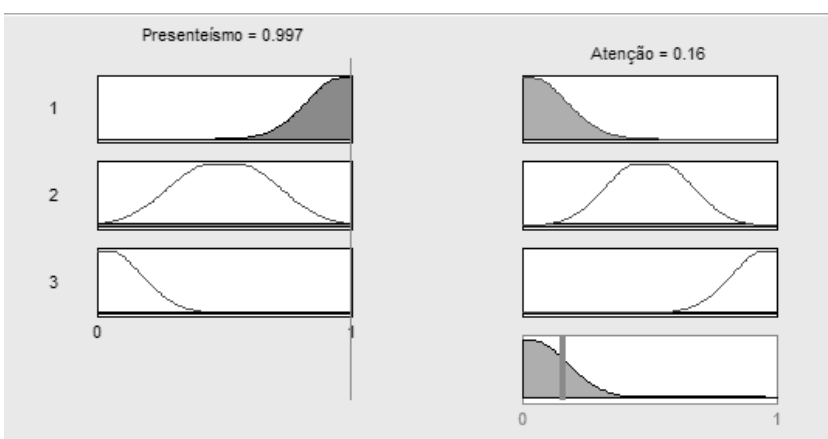

Fonte: Elaborada pelos autores

Mediante a atribuição da função de pertinência Gaussiana para as variáveis que aborda, este caso apresenta um modelo que permite explicitar e mensurar, com valores entre 0 e 1 , a relação inversamente proporcional que se estabelece entre $o$ comportamento presenteísta e o índice de atenção individual no trabalho. Observa-se que o nível de atenção decresce na medida em que se aumenta a propensão deste comportamento, podendo atingir um lapso de atenção de aproximadamente $68,2 \%$.

Assim, sabendo que a atenção consiste em um fator que se relaciona diretamente com a produtividade, a qualidade e a aprendizagem - limitando o desempenho em quantidade, qualidade e em méritos de capacitação - este resultado é um indicativo do teor de impacto do presenteísmo nos resultados da organização.

A representatividade do que é constatado por meio do modelo se apresenta como uma ressalva acerca da importância de serem consolidadas estratégias de amenização deste fenômeno que ameaça a sustentabilidade organizacional.

Para o Caso $2^{\circ}$, o nível da variável Atenção é apresentado na Figura 5 e os níveis das variáveis de saída, Aprendizagem, Produtividade e Qualidade, são apresentados na Figura 6.

Figura 5 . Nível da variável Atenção

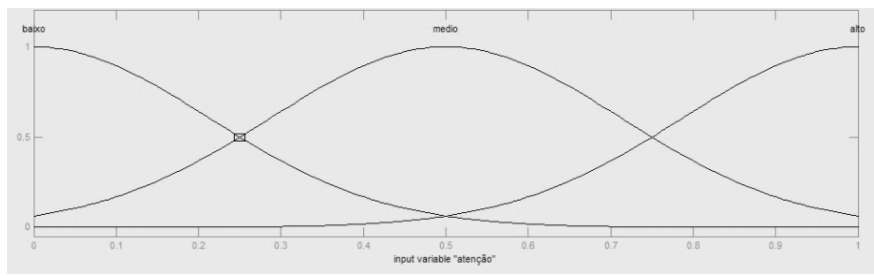

Fonte: Elaborada pelos autores 
Figura 6 . Níveis das variáveis Aprendizagem, Produtividade e Qualidade

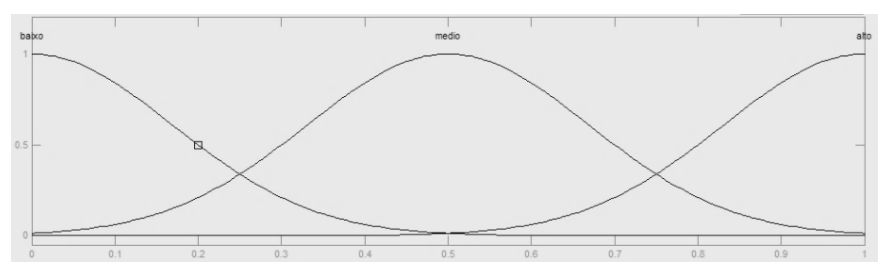

Fonte: Elaborada pelos autores

Observa-se que os pontos de mínimo e de máximo global e local da função estão em torno de $(0,0.203)$ e $(1,0.797)$. Ou seja, se a variável Atenção for baixa, as variáveis Aprendizagem, Produtividade e Qualidade serão mínimas, em torno de 20,3\%. Em contrapartida, se a Atenção estiver no seu valor máximo igual a 1 , as variáveis Aprendizagem, Produtividade e Qualidade terão seus valores máximos em torno de 79,7\%.

A visão geral do comportamento e da variação das variáveis estudadas no Caso $2^{\circ}$ é exposta na Figura 7, na qual se pode observar o comportamento das variáveis Aprendizagem, Produtividade e Qualidade em relação aos valores de mínimo e de máximo da variável Atenção.

Figura 7 - Gráficos de saída das variáveis Aprendizagem, Produtividade e Qualidade

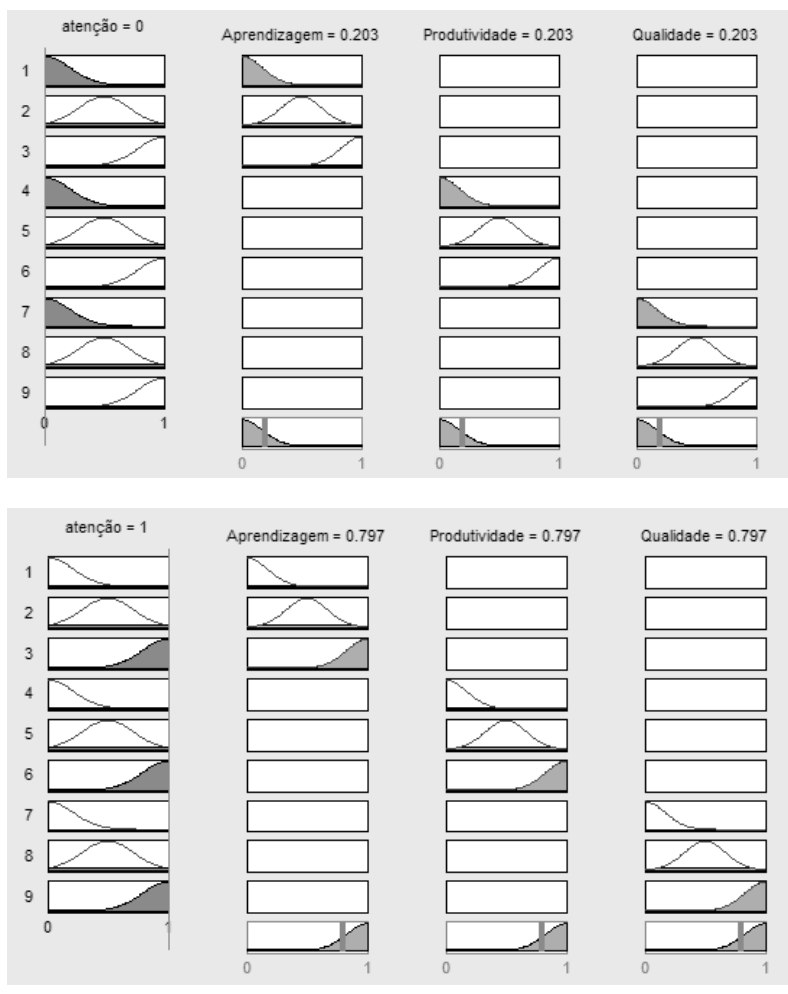

Fonte: Elaborada pelos autores
Este segundo caso explicita uma maneira de mensurar a relação diretamente proporcional que se estabelece entre o nível de atenção do trabalhador e o desempenho no trabalho. Pode-se observar que a eficiência e a eficácia do indivíduo no trabalho, bem como a sua capacidade para aprender, decrescem mediante a diminuição do nível de atenção.

Como se pode notar, o envolvimento em nãotrabalho no trabalho gera lapsos no nível de atenção do indivíduo que podem limitar o rendimento físico e mental, aumentar a incidência de retrabalhos e atenuar a propensão individual ao desenvolvimento de novos conhecimentos e habilidades, em uma proporção de aproximadamente 59,4\%.

Este resultado confirma o exposto no tópico 3.3 de que os efeitos do presenté́smo no resultado organizacional são delimitados pelo seu impacto no índice de atenção individual. Pelos lapsos de atenção que gera, pode-se considerar que o presenteísmo limita o desempenho individual no trabalho - em quantidade, em qualidade e em méritos de capacitação - em mais da metade.

Neste ponto, vale resgatar o mencionado no tópico 3.2 acerca das ligações entre o comportamento presenteísta e o significado que o indivíduo atribui ao trabalho. Do que é exposto neste caso, não há dúvida de que a delimitação do impacto do comportamento presenteísta na eficiência e eficácia do trabalho e, consequentemente, no resultado organizacional, é atrelada ao quanto o trabalho é significativo àquele que o executa. Sendo dotado de sentido, o trabalho, por si próprio, suscita a atenção do indivíduo, despertando-o ao aprendizado e direcionando-o a melhores resultados.

Já em relação ao Caso $3^{\circ}$, as Figuras 8 e 9 demonstram a forma como a Qualidade de Vida e a Cooperação interferem no Presenteísmo:

Figura 8 . Níveis das variáveis Qualidade de Vida e a Cooperação

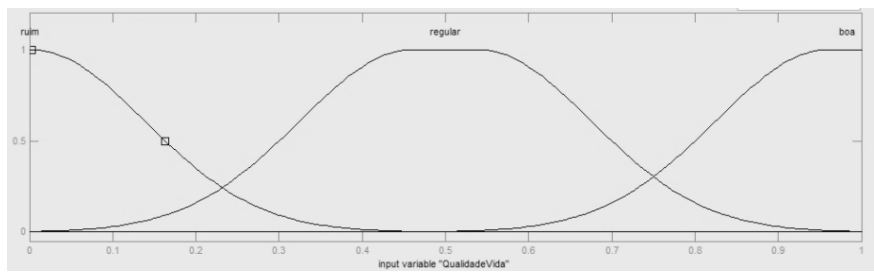




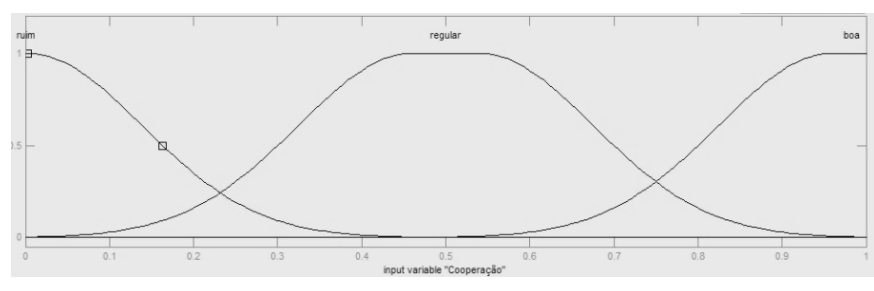

Fonte: Elaborada pelos autores

Figura 9 . Nível da variável Presenteísmo

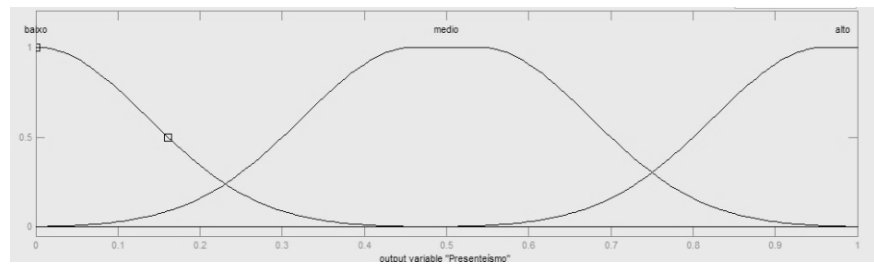

Fonte: Elaborada pelos autores

Pode-se observar que os pontos de mínimo da função estão em $(0,0.874)$. Isso significa que se a Qualidade de Vida e a Cooperação forem nulas o valor do Presenteísmo será em torno de $87,4 \%$. Já os pontos de máximo estão em (1, 0.112). Ou seja, se a Qualidade de Vida e a Cooperação forem máximas, o valor do Presenteísmo será mínimo, em torno de $11,2 \%$.

A Figuras 10 expõe a visão geral do comportamento e da variação das variáveis estudadas no Caso $3^{\circ}$, podendo-se observar o comportamento da variável Presenteísmo com relação aos valores de mínimo e de máximo das variáveis Qualidade de Vida e Cooperação.

Figura 10 . Gráficos de saída das variáveis Qualidade de Vida, Cooperação e Presenteísmo

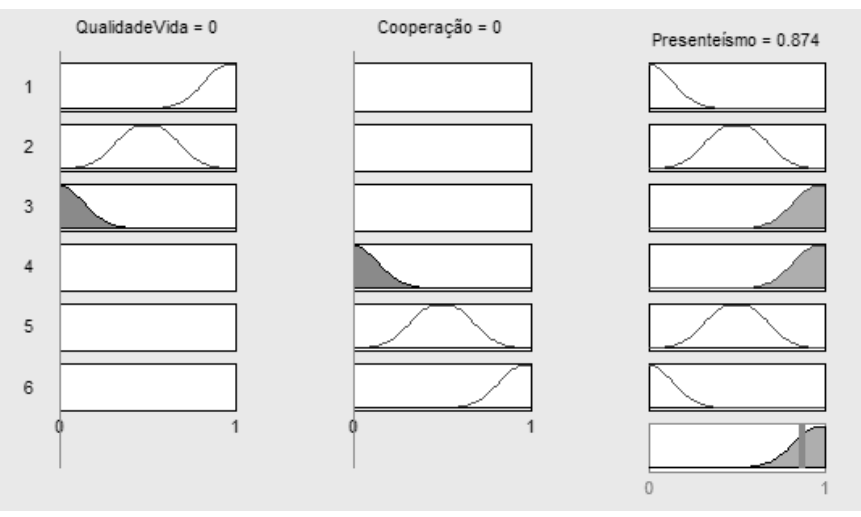

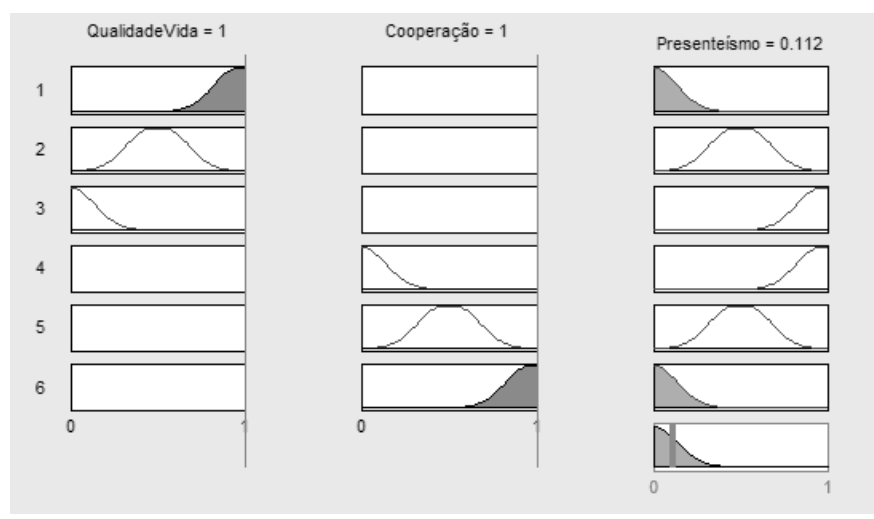

Fonte: Elaborada pelos autores

Este caso quantifica a relação inversamente proporcional entre o Presenteísmo e seus dois principais determinantes organizacionais: Cooperação e Qualidade de Vida. O resultado constatado pelo modelo é um demonstrativo de que condições de trabalho e níveis de cooperação favoráveis são potenciais para diminuir a propensão individual em apropriar o tempo de trabalho com não-trabalho. Segundo o que exposto, pode-se afirmar que se os indicadores físicos e psicológicos de bem estar e as atitudes cooperativas da empresa estiverem em seu nível máximo de favorabilidade, o ato de ausentar-se em mente ou comportamento do trabalho pode ser reduzido em até $76,2 \%$.

Pode-se considerar que os resultados deste modelo justificam o teor analítico proposto pelo estudo na abordagem do presenteísmo. Tendo em vista a representatividade da influência destes fatores no fenômeno abordado, o que é exposto neste modelo vai de encontro às vertentes majoritárias que insistem em enviesar as análises do presenteísmo estritamente a fatores pessoais. Observa-se que os fatores organizacionais exercem influência representativa na manifestação deste comportamento e, portanto, devem ser considerados no estudo das causas e consequências concernentes a ele, bem como na busca pela consolidação de estratégicas que amenizem seus impactos na sustentabilidade organizacional.

Diante do conjunto de variáveis abordadas e da análise dos dados que as envolvem, pode-se fazer a combinação delas obtendo as seguintes regras para o Caso $1^{\circ}$ :

1) Se (Presenteísmo é alto) então (Atenção é baixa);

2) Se (Presenteísmo é médio) então (Atenção é média); 
3) Se (Presenteísmo é baixo) então (Atenção é alta);

Para o Caso $2^{\circ}$ foram geradas as seguintes regras:

1) Se (Atenção é baixa) então (Aprendizagem é baixa);

2) Se (Atenção é média) então (Aprendizagem é média);

3) Se (Atenção é alta) então (Aprendiragem é alta);

4) Se (Atenção é baixa) então (Produtividade é baixa);

5) Se (Atenção é média) então (Produtividade é média);

6) Se (Atenção é alta) então (Produtividade é alta);

7) Se (Atenção é baixa) então (Qualidade é baixa);

8) Se (Atenção é média) então (Qualidade é média);

9) Se (Atenção é alta) então (Qualidade é alta);

Por fim, para o Caso $3^{\circ}$, tem-se:

1) Se (Qualidade de Vida é boa) então (Presenteísmo é baixo);

2) Se (Qualidade de Vida é regular) então (Presenteísmo é médio);

3) Se (Qualidade de Vida é ruim) então (Presenteísmo é alto);

4) Se (Cooperação é ruim) então (Presenteísmo é alto);

5) Se (Cooperação é regular) então (Presenteísmo é médio);

6) Se (Cooperação é boa) então (Presenteísmo é baixo);

Em suma, mediante a base de regras e os modelos gerados, nota-se que o modelo permite obter para cada valor dado às variáveis de entrada (Presenteísmo, Atenção, Qualidade de Vida e Cooperação) um valor diferente para as variáveis de saída (Atenção, Aprendizagem, Produtividade, Qualidade e Presenteísmo), possibilitando a automatização do mapa sistêmico que compõem.

Com isso, ao possibilitar a previsão do comportamento das variáveis que circundam o presenteísmo e a mensuração das relações entre elas, este modelo Fuzzy é potencial em subsidiar as decisões da gestão e servir de orientação para as estratégias de amenização deste fenômeno que ameaça a sustentabilidade organizacional.

\section{Conclusão}

Este estudo teve como tema central um dos principais problemas relacionados ao capital humano: o presenteísmo. Seu desenvolvimento permitiu considerar que, em termos conceituais, as análises predominantes acerca do presenteísmo limitamse a relacioná-lo apenas aos aspectos da saúde do trabalhador. Assim, a relevância desta pesquisa residiu no caráter diferenciado da reflexão que promove, analisando-o como algo não relacionado estritamente à doença, mas como algo crônico. um comportamento que não é estritamente relacionado à saúde do trabalhador, mas que pode assumir um caráter crônico, independentemente da condição física daquele que o manifesta

Por estas razões, a análise conceitual do presenteísmo foi estruturada a partir de sua acepção de não-trabalho no trabalho, relacionando a sua manifestação ao uso desequilibrado do tempo no trabalho com aquilo que não é relacionado ao trabalho, daí seu impacto na produtividade. Considerando sua ocorrência na interface entre fatores pessoais e organizacionais, considerou-se que o comportamento presenteísta pode ser determinado por uma propensão individual voluntária ou pela própria irracionalidade da organização.

Assim, sendo considerado como o resultado de uma combinação complexa de fatores organizacionais e humanos, este estudo propôs-se a abordar o presenteísmo em consonância ao atual desafio da gestão de identificar e mensurar este comportamento e seus impactos para o desempenho competitivo da empresa, recorrendo-se, para tanto, à Lógica Fuzzy. Tratando-se de um comportamento crônico que ameaça a sustentabilidade organizacional em todos os seus aspectos, considerou-se o fato de abordá-lo por meio de uma lógica matemática um caminho potencial em relevância científica e organizacional.

Desta forma, fundamentando-se na construção de um mapa sistêmico, que inclui as principais variáveis que circundam o construto presenteísmo, este estudo teve como objetivo apresentar um modelo matemático Fuzzy para mensurar as relações entre elas e automatizar tal representação sistêmica. 
A justificativa para o que foi proposto refere-se ao fato de o modelo permitir a combinação e a previsão do comportamento das variáveis de interesse. Pelo fato da Lógica Fuzzy trabalhar com variáveis contidas no cotidiano do trabalhador, as soluções geradas pelo modelo se tornam mais próximas da realidade do que os obtidos pela Lógica Clássica, possibilitando identificar aspectos ainda não questionados e delimitar inferências acerca do comportamento presenteísta que vão além do que é possível observar por meio de técnicas básicas e tradicionais.

Deste modo, o modelo abordado se porta como um subsídio quantitativo para a gestão do presenteísmo. Sabe-se que, frente ao atual dinamismo do mercado, a intervenção do gestor é necessária para se obter melhores resultados na empresa, tornando-se evidente a necessidade de utilização de ferramentas que auxiliem nas tomadas de decisão. Deste modo, a utilidade do modelo Fuzzy proposto para o estudo do presenteísmo se deve ao fato de possibilitar a leitura de cada uma das variáveis qualitativas de entrada em uma variável de saída quantitativa, garantindo rigor às decisões relacionadas e assertividade à gestão deste comportamento. Afinal, uma gestão quantitativa se porta como uma estratégia imune às decisões sujeitas a vieses subjetivados.

Além disso, o modelo Fuzzy proposto permite manutenções periódicas das variáveis utilizadas, possibilitando seu ajustamento de acordo com as necessidades da empresa. Isso faz com que todas as soluções apresentadas possam ser integradas com as diferentes áreas da organização. Permitindo elencar os valores das variáveis de entrada em tempo real, o monitoramento constante do modelo é viabilizado, possibilitando mantê-lo na forma desejada para a equipe e para a empresa.

Mediante essas justificativas apresentadas, é válido destacar que o objetivo de apresentar um modelo para mensurar as relações que circundam o presenteísmo e automatizar o mapa que sistemicamente o representa não é atrelado ao intuito de contribuir para a propagação de uma ideologia gerencial que defenda a total aderência do indivíduo ao trabalho e a necessidade de que ele se doe inteiramente para que a empresa possa explorá-lo o máximo possível e auferir cada vez mais ganhos econômicos. O fato de não alinhar o estudo do comportamento apenas aos fatores pessoais, mas também atribuir sua manifestação ao caráter pouco humanista das empresas que, muitas das vezes, o propicia, já foi uma forma de enfatizar que, ao contrário do que pode parecer, a abordagem do estudo não se apresenta como um reforço à alienação individual ao trabalho, mas ao seu próprio combate. Considera-se que uma abordagem Fuzzy potencial em subsidiar decisões estratégicas de amenização de um dos mais atuais problemas relacionados ao capital humano não é um reforço à mais valia e à alienação do indivíduo; o comportamento presenteísta consiste na atual característica de um indivíduo que já é alienado.

Há de se considerar que este artigo pode ser subsídio para novos estudos. O mapa sistêmico, com suas variáveis direta ou inversamente relacionadas, sugere sistemas de medição para essas grandezas no contexto organizacional onde a produção realmente ocorre.

Além disso, os resultados do estudo foram sustentados por inferências lógicas que são passíveis de consideração em estudos posteriores que visem a sua aplicação a uma amostra de indivíduos na empresa, de modo a corroborar ou não os resultados. Da mesma forma, pelo viés da modelagem matemática, pode-se mencionar a possibilidade de testar o modelo proposto em um banco de dados de modo que se possa comprovar a sua acurácia e direcionar a criação de novos parâmetros para que o torne ainda mais eficaz.

Ainda, a abordagem ao presenteísmo limitou-se à questão do não-trabalho no trabalho, não sendo consideradas outras possíveis abordagens capazes de também contribuir com novos lampejos acerca do comportamento, abrindo-se, portanto, possibilidades de novas reflexões sobre o construto.

\section{Referências bibliográficas}

\section{BARROS, L.; BASSANEZI, R. C. Tópicos de lógica fuzzy e biomatemática. Unicamp, 2010.}

BEDE, B.; STEFANINI, L. Generalized differentiability of fuzzy-valued functions. Fuzzy Sets and Systems, v.230, p.119-141, 2013.

BÖCKERMAN, P.; LAUKKANEN, E., Predictors of sickness absence and presenteeism: does the pattern differ by a respondent's health? Journal of Occupational and Environmental Medicine, v.52, n.3, p.332-335, 2010.

What makes you work while you are sick?

Evidence from a survey of workers. European Journal of Public Health, v.20, n1, p.43-46, 2009. 
COLLINS, A.; CARTWRIGHT, S. Why come into work ill? Individual and organizational factor underlying presenteeism. Employee Relation, v.34, n.4, p.429-442, 2012.

CONTU, A. Decaf resistance on misbehavior, cynicism, and desire in liberal workplaces.

Management Communication Quarterly, v.21, n.3, p.364-379, 2008.

COX, E. Fuzzy logic for business and industry. Massachusetts: Charles River Media Inc, 1995.

D'ABATE, C.P. Working hard or hardly working: a study of individuals engaging in personal business on the job. Human Relations, v.58, n.8, p.1009-1032, 2005.

D'ABATE, C.P.; EDDY, E.R. Engaging in personal business on the job: extending the presenteeism construct. Human Resource Development Quarterly, v.18, n.3, p.361-383, 2007.

EDDY, E.R.; D'ABATE, C.P.; THURSTON JR, P.W. Explaining engagement in personal activities on company time. Personnel Review, v.39, n.5, p.639354, 2010.

FERREIRA, A.I. et al. LMX as a negative predictor of presenteeism climate: a cross-cultural study in the financial and health sectors. Journal of Organizational Effectiveness: People and Performance, v.2, n.3, p.282-302, 2015.

FERREIRA, A.I.; ESTEVES, J.D. Perceptions of time at work. Personnel Review, v.45, n.1, p.29-50, 2016.

FRITZ, C. et al. Embracing work breaks: recovering from work stress. Organizational Dynamics, v.42, p.274-280, 2013.

FRITZ, C.; LAM, C.F.; SPREITZER, G.M. It's the little things that matter: an examination of knowledge workers' energy management. The Academy of Management Perspectives, v.25, n.3, p.28-39, 2011.

GABRIEL F.; LUÍS R. A., et al. Sistema baseado em regras fuzzy para classificação de mão de obra em relação ao grau de aptidão na utilização de colheitadeiras de cana no setor sucroalcooleiro. II CONGRESSO BRASILEIRO DE SISTEMAS FUZZY, 2. Anais II CBSF, 2012.
GUPTA, M.M.; KAUFMANN, A. Fuzzy

mathematical models in engineering and

management science. Amsterdam: Elsevier Science Publishers B, 1988.

IVARSSON, L.; LARSSOM, P. Personal activities on company time to make everyday life work. In: BERGMAN, A.; HUZELL, B. Segregationens seghet och dess föränderliga former. Karlstad, Sweden: Universitetstryckeriet, 2012. p.127-137.

MOREIRA, F.M.; BAPTISTA, R.D. As novas tecnologias produtivas e as mudanças nas competências individuais. In: V Congresso de Administração e Contabilidade da UFV,

2012, Viçosa. Anais do V Congresso de Administração e Contabilidade da UFV, 2012.

PAGE, D. Teacher's personal web use at work. Behavior \& Information Technology, v.34, n.5, p.443-453, 2015.

PAULSEN, R. Layers of dissent: the meaning of time appropriation. Outlines. Critical Practice Studies, v.13, n.1, p.53-81, 2011.

PAULSEN, R., Now-work at work: resistance or what? Organization, v.22, n.3, p.351-367, 2015.

SAARVALA, E. Presenteeism: the latest attack on economic and human productivity. Human Resources Management, 2006.

SILVEIRA, A. M. et al. Identificação de abordagens administrativas: Um ensaio com lógica fuzzy.

INFOCOMP Journal of Computer Science 4.1, p. 36-45, 2004.

SILVEIRA, M. A. et al. Inovação e aprendizagem organizacional: abordagem TCD para

desenvolvimento de competências em empresa do setor eletrônico. In: XVI Congresso LatinoIberoamericano de Gestão de Tecnologia, 2015, Porto Alegre. Anais do XVI Congresso LatinoIberoamericano de Gestão de Tecnologia, 2015.

SILVEIRA, M. A.; BECARO, T. C. Competitividade com qualidade de vida: estratégias e práticas baseadas na valorização do fator humano nas organizações. In: SILVEIRA, M. A; BECARO, T. C. (Orgs.). Competitividade com qualidade de vida: o capital humano como fator de produção. Campinas, SP: CTI (Centro de Tecnologia da Informação Renato Archer), 2014. p.21-37. 
SHAW, I. S.; SIMÕES, M. G. Controle e

modelagem fuzzy. São Paulo: Edgard Blucher, 1999.

TALOYAN, M. et al. Sickness presenteeism predicts suboptimal self-rated health and sickness absence: a nationally representative study of the Swedish working population. PLOS ONE, v. 7, n. 9, 2012. Disponível em $<$ http://bit.ly/1QO6kBh $>$. Acesso em 02 mai. 2016.

TROUGAKOS, J.P.; HIDEG, I. Momentary work recovery: the role of within-day work breaks.

Current perspectives on job-stress recovery:

research in occupational stress and well being, v. 7, p. 37-84, 2009.

UMANN, J.; GUIDO, L. de A.; GRAZZIANO, E. da S. Presenteísmo em enfermeiros hospitalares. Rev. Latino-Am. Enfermagem, Ribeirão Preto, v. 20, n. 1, 2012.

UMANN, J.; GUIDO, L. de A.; SILVA, R.M. da. Estresse, coping, e presenteísmo em enfermeiros que assistem pacientes críticos e potencialmente críticos. Rev Esc Enferm USP, São Paulo, v. 48, n. 5, p. 891898, 2014. Disponível em: <http://bit.ly/1rejXVz>. Acesso em: 02 mai. 2016.

VIJAYAKUMAR, P.B. Work-life balance, identity salience and break taking behaviors in Indian expatriates. 2015, 83p. Dissertação (Master of Science: Psychology), The University of Tennessee at Chattanooga. Chattanooga, Tennessee, 2015.

YEN, J.; LANGARI, R.; ZADETH, L. A. Industrial applications of fuzzy logic and intelligent systems. New York: IEEE Press, 1994.

ZADEH, L.A. Fuzzy Sets-Inform. and Control, n. 8, p. 338-353, 1965. 